\title{
Paediatric crossword puzzle - 23 (Answers)
}

Manouri P. Senanayake ${ }^{1}$

Sri Lanka Journal of Paediatrics, 2014; 43(4): 184

\begin{tabular}{|r|l|l|l|l|l|l|l|l|l|l|l|l|l|}
\hline${ }^{1} \mathrm{C}$ & $\mathrm{O}$ & $\mathrm{O}$ & $\mathrm{L}$ & & ${ }^{2} \mathrm{~F}$ & $\mathrm{E}$ & $\mathrm{D}$ & $\mathrm{E}$ & $\mathrm{R}$ & $\mathrm{M}$ & ${ }^{3} \mathrm{~A}$ & $\mathrm{~N}$ & ${ }^{4} \mathrm{~S}$ \\
\hline $\mathrm{R}$ & & & & & & & & & & & $\mathrm{C}$ & & $\mathrm{Y}$ \\
\hline${ }^{5} \mathrm{~F}$ & ${ }^{6} \mathrm{R}$ & $\mathrm{A}$ & ${ }^{7} \mathrm{G}$ & $\mathrm{M}$ & $\mathrm{E}$ & ${ }^{8} \mathrm{~N}$ & $\mathrm{~T}$ & ${ }^{9} \mathrm{E}$ & $\mathrm{D}$ & & $\mathrm{H}$ & & $\mathrm{N}$ \\
\hline & $\mathrm{A}$ & & $\mathrm{I}$ & & & $\mathrm{O}$ & & $\mathrm{M}$ & & & $\mathrm{O}$ & & $\mathrm{D}$ \\
\hline & $\mathrm{N}$ & & $\mathrm{B}$ & & & $\mathrm{D}$ & & $\mathrm{P}$ & & & $\mathrm{N}$ & & $\mathrm{A}$ \\
\hline & $\mathrm{U}$ & & $\mathrm{B}$ & & ${ }^{10} \mathrm{~W}$ & & & ${ }^{11} \mathrm{H}$ & $\mathrm{E}$ & $\mathrm{R}$ & $\mathrm{D}$ & & $\mathrm{C}$ \\
\hline & $\mathrm{L}$ & & $\mathrm{U}$ & & ${ }^{12} \mathrm{P}$ & $\mathrm{L}$ & $\mathrm{A}$ & ${ }^{13} \mathrm{Y}$ & & & & ${ }^{14} \mathrm{~V}$ & $\mathrm{~T}$ \\
\hline & $\mathrm{A}$ & & $\mathrm{S}$ & & $\mathrm{W}$ & & & ${ }^{15} \mathrm{~S}$ & $\mathrm{C}$ & $\mathrm{U}$ & $\mathrm{R}$ & $\mathrm{V}$ & $\mathrm{Y}$ \\
\hline${ }^{16} \mathrm{R}$ & & & & ${ }^{17} \mathrm{~T}$ & & ${ }^{18} \mathrm{E}$ & & $\mathrm{E}$ & & & & ${ }^{19} \mathrm{M}$ & $\mathrm{L}$ \\
\hline $\mathrm{E}$ & & & & $\mathrm{A}$ & & $\mathrm{P}$ & & $\mathrm{M}$ & & ${ }^{20} \mathrm{~F}$ & & & $\mathrm{Y}$ \\
\hline${ }^{21} \mathrm{D}$ & $\mathrm{E}$ & ${ }^{22} \mathrm{C}$ & $\mathrm{O}$ & $\mathrm{R}$ & $\mathrm{T}$ & $\mathrm{I}$ & ${ }^{23} \mathrm{C}$ & $\mathrm{A}$ & $\mathrm{T}$ & $\mathrm{E}$ & & & \\
\hline & & $\mathrm{O}$ & & $\mathrm{G}$ & & & $\mathrm{F}$ & & & $\mathrm{T}$ & & & \\
\hline & ${ }^{24} \mathrm{U}$ & $\mathrm{L}$ & $\mathrm{C}$ & $\mathrm{E}$ & $\mathrm{R}$ & & & & ${ }^{25} \mathrm{~B}$ & $\mathrm{U}$ & $\mathrm{L}$ & $\mathrm{L}$ & $\mathrm{A}$ \\
\hline & & $\mathrm{D}$ & & ${ }^{26} \mathrm{~T}$ & $\mathrm{I}$ & $\mathrm{G}$ & $\mathrm{E}$ & $\mathrm{R}$ & & $\mathrm{S}$ & & & \\
\hline
\end{tabular}

${ }^{1}$ Senior Professor in Paediatrics, Faculty of Medicine, University of Colombo 